\title{
INTERACCIÓN ENTRE EL PERSONAL DE SALUD Y LAS JÓVENES EMBARAZADAS DURANTE EL CONTROL PRENATAL: UN ESTUDIO CUALITATIVO*
}

\author{
Carolina Blossiers ${ }^{1}$
}

\begin{abstract}
RESUMEN
Objetivo. Explorar la interacción entre el personal de salud y las adolescentes gestantes, a partir de los significados de la sexualidad, el embarazo y el control prenatal en un Hospital Nacional de Lima. Materiales y métodos. Se realizó un estudio cualitativo que incluyó observaciones de la interacción durante la consulta, entrevistas a profundidad a gestantes adolescentes (14) y personal de salud (9) y un grupo focal de gestantes. Resultados. Existen percepciones y valoraciones socioculturales que no favorecen la comunicación entre ambos. El personal de salud argumenta que tener experiencias sexuales precoces significa que las jóvenes asumen el papel de adultas; consideran que las jóvenes no estaban preparadas biopsicológica y socialmente para ser madres. En esta concepción, el cuerpo de las jóvenes es concebido como un medio de control, intervención y poder ante la presencia del ginecoobstetra. Sin embargo, para las jóvenes embarazadas representa valorar su cuerpo como futura madre. Los significados del control prenatal para el personal de salud, se orientan hacia los aspectos biomédicos desde el enfoque de riesgo; enfatizando en el contagio de enfermedades de transmisión sexual; en cambio para las adolescentes, es importante el trato por el personal de salud, que es diferenciado, las mujeres ofrecen un trato acogedor y cálido, sin descuidar los aspectos cognitivos y científicos; mientras que los varones, en la mayoría de los casos, tienen una interacción más operativa y cognitiva. Conclusión. La interacción entre el personal de salud y las jóvenes embarazadas varía según el sexo del personal de salud y tiene significados diferentes para las gestantes, por lo que debe tenerse en cuenta esta información para mejorar la atención en el control prenatal de las adolescentes.
\end{abstract}

Palabras clave: Embarazo en adolescencia; Personal de salud; Relaciones profesional-paciente; Atención prenatal (fuente: DeCS BIREME).

\section{INTERACTION BETWEEN HEALTH PERSONNEL AND YOUNG PREGNANT WOMEN DURING PRENATAL CONTROL: A QUALITATIVE STUDY}

\begin{abstract}
Objective. To explore the interaction between health personnel and young pregnants, beginning from the meanings of sexuality, pregnancy and prenatal control in a Lima National Hospital. Materials and methods. A qualitative study was conducted, including observations of the interaction during the consult, in-depth interviews to young pregnants (14) and health personnel (9), and a focus group of young pregnant women. Results. There are perceptions and socio-cultural valuations that don't favor communication between both groups. Health personnel rise as an argument the fact that having prompt sexual relations means that the youngsters assume the role of adults, and also consider that these weren't prepared bio-psicologically and socially to be mothers. In this conception, the body of the youngster is considered a means of control, intervention and power in the presence of the obstetrician. Nevertheless, for the pregnant youngsters it represents its value as future mothers. The meanings of the prenatal control for the health personnel are oriented to the biomedical aspects from the risk approach; emphasizing in the transmission of sexually transmitted diseases; on the other hand, for the teens the behavior of the health staff is important and differs, women offer a kind, warm attention, without overlooking the scientific and cognitive aspects, while males, in most of the cases, have a more operative and cognitive interaction. Conclusion. The interaction between health personnel and pregnant women varies according to the gender of the health staff and has different meanings for the pregnant, which is important to take into account in order to improve the attention in prenatal control of young women.
\end{abstract}

Key words: Pregnancy in adolescence; Health personnel; Professional-patient relations; Prenatal care (source: MeSH $N L M)$.

\footnotetext{
Socióloga especializada en salud. Maestra en Género, Sexualidad y Salud Reproductiva. Facultad de Salud Pública, Universidad Peruana Cayetano Heredia. Lima, Perú.

* Los resultados de esta investigación son parte de la tesis: Blossiers C. Significados de la interacción entre el personal de salud y las jóvenes durante el control prenatal en un hospital público de Lima. Lima: Universidad Peruana Cayetano Heredia; 2007.
}

Recibido: 16-06-09 Aprobado: 11-08-10 


\section{INTRODUCCIÓN}

El embarazo en la adolescencia es un tema de interés en salud pública y bastante abordado desde la demografía y ciencia biomédica, a partir del crecimiento de la fecundidad y el enfoque de riesgo (1-3), aunque menos desde la comprensión de los factores socioculturales, que aquí se abordan.

En el Perú y otros países en desarrollo existen elevados índices de mortalidad materna ${ }^{(3,4)}$. En ese sentido los gobiernos desarrollan diferentes estrategias para mejorar la atención de la salud de la embarazada $(1,5)$. Hay consenso en que el control prenatal, busca determinar las condiciones de inicio del embarazo, posibles complicaciones, evaluar el crecimiento uterino y la evolución del feto ${ }^{(5,6)}$. Recomendar normas básicas para la alimentación, nutrición del bebé y la embarazada, para reducir así las complicaciones obstétricas y las altas tasas de mortalidad ${ }^{(5-7)}$.

Teniendo en cuenta lo señalado, existen referencias donde se señala que el acceso al control prenatal ha contribuido al descenso de la mortalidad materna, en general en el país se ha disminuido en 80 muertes por 100 mil nacidos vivos entre 1996 y el $200{ }^{\left({ }^{(8)}\right.}$.

En ese sentido, según el Ministerio de Salud, entre 1992 a 1997, el Programa Materno Perinatal ha incrementado en forma relevante la cobertura de la atención prenatal; el número de controles prenatales atendidos creció de 307255 a 524946 estimándose un total de 563856 controles en $1998^{(8)}$.

A su vez, estudios previos han señalado la importancia de garantizar el acceso y la calidad de atención en el control prenatal para promover la salud de la madre y el bebé ${ }^{(5,9)}$; se ha implementado en el país programas, como el Seguro Integral de Salud (SIS), que no logran satisfacer las necesidades y expectativas relacionadas con la falta de información, el trato recibido y atención a las condiciones de salud durante el control prenatal ${ }^{(8)}$.

Por ello, el propósito del estudio es conocer la interacción entre el personal de salud y la joven embarazada, partiendo desde las percepciones sobre el trato y la atención y, de esta forma, considerar que existen conceptos o valoraciones socioculturales que preceden a la relación social ${ }^{(10)}$, y que a la vez estos conceptos muchas veces pueden ser una barrera o facilitar la comunicación entre el personal de salud y las jóvenes embarazadas, durante el control prenatal. Todo ello en función a la interpretación de los significados de la sexualidad, el embarazo y la atención prenatal que fueron definiendo la interacción en el servicio de ginecoobstetricia general, del adolescente y psicoprofilaxis obstétrica.

\section{MATERIALES Y MÉTODOS}

\section{DISEÑO Y ÁREA DE ESTUDIO}

Se realizó un estudio cualitativo en un hospital nacional de Lima durante el año 2007. La atención prenatal de las jóvenes gestantes se da en el marco institucional del Seguro Integral de Salud (SIS) del Ministerio de Salud. Todas provenían de sectores marginales, y tuvieron la oportunidad de recibir la visita de una asistenta social a sus domicilios para evaluar su situación socioeconómica, afiliarse a este programa y tener derecho a recibir atención ginecoobstétrica en el centro de salud y el hospital de su jurisdicción.

Las jóvenes embarazadas se presentaron al servicio de admisión del hospital, luego se dirigieron al servicio de triaje con la obstetriz para registrar antecedentes clínicos como: el peso, la talla uterina y la presión arterial. En seguida ingresaron al servicio de ginecoobstetricia general y del adolescente (Figura 1). En estas citas el ginecoobstetra evaluaba los riesgos de ETS/VIH-SIDA, el tamaño de la pelvis para el parto normal y realizaba el examen dePapanicolaou. Finalmente, fueron transferidas al consultorio de la especialidad de ginecoobstetricia del adolescente, para que los médicos detecten posibles complicaciones del embarazo, previniendo los riesgos en la gestación y realizando vigilancia al crecimiento del bebé.

\section{POBLACIÓN DE ESTUDIO}

Se trabajó con dos poblaciones, jóvenes embarazadas y personal de salud. Las jóvenes embarazadas fueron seleccionadas por conveniencia -hasta tener saturación de la información- a partir del reporte de atenciones del servicio de triaje, antes que ingresaran a los servicios de ginecoobstetricia general y del adolescente; se incluyó a aquellas entre 15 y 19 años, primerizas, entre el sexto y noveno mes de gestación y de sectores pobres; sus características se muestran en la Tabla 1.

El personal de salud fue seleccionado por conveniencia, hasta tener saturación de la información; se incluyó tres médicos ginecólogos y tres obstetrices (ambos grupos con dos o más años en trabajo con adolescentes) y dos médicos residentes de ginecología, el rango de edades estuvo entre los 30 a 48 años, $3 / 9$ son casados y 6/9 son mujeres. 


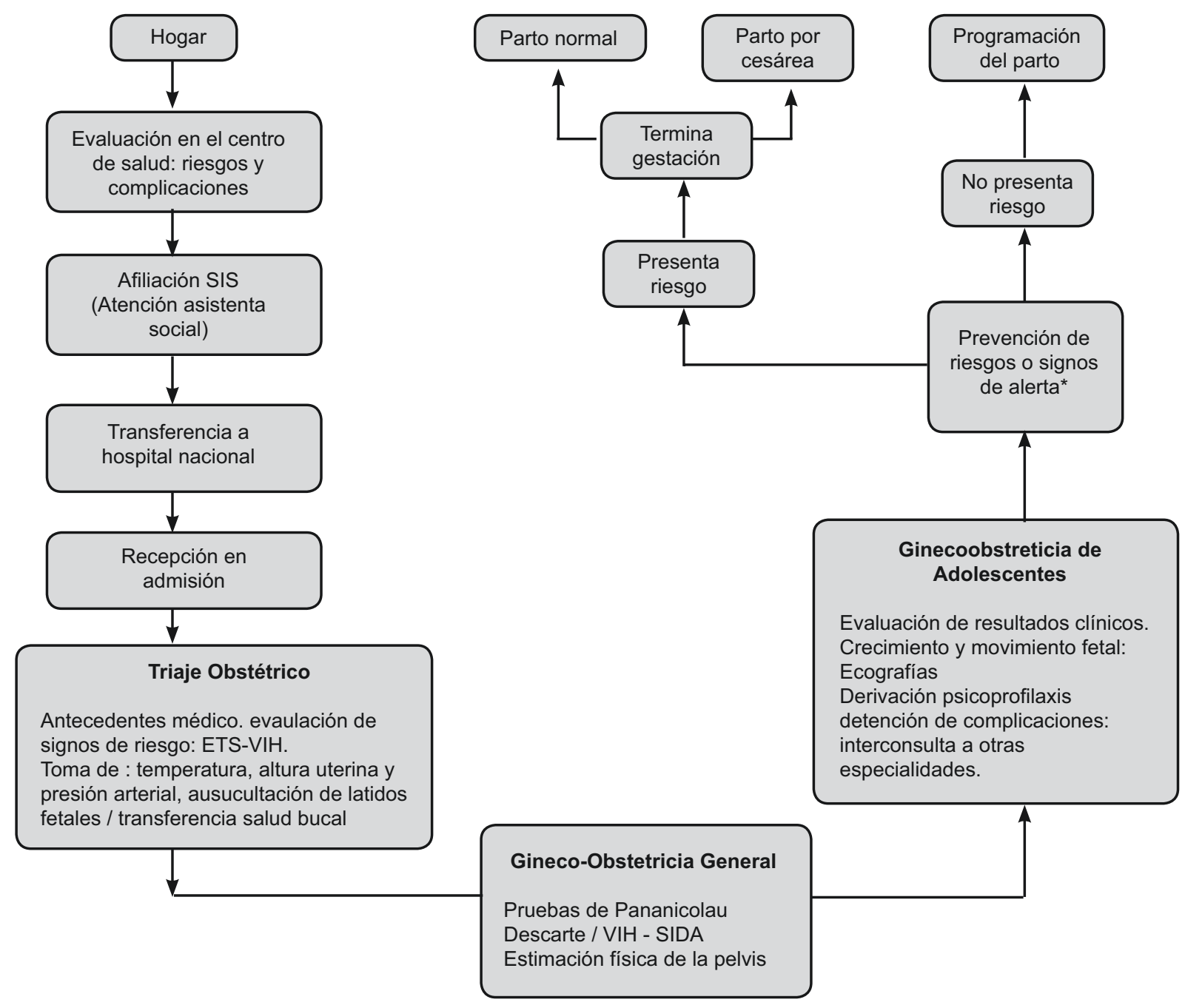

Figura 1. Flujograma de asistencia al control prenatal de las jóvenes embarazadas.

* Sangrado, contracciones uterinas lentas, ausencia de movimientos fetales, hinchazón de pies, cefalea y elevación de presión arterial.

Tabla 1. Características de las jóvenes embarazadas primigestas.

\begin{tabular}{ccccc}
\hline Edad & $\begin{array}{c}\text { Grado de } \\
\text { instrucción }\end{array}$ & $\begin{array}{c}\text { Estado } \\
\text { civil }\end{array}$ & $\begin{array}{c}\text { Lugar de } \\
\text { nacimiento }\end{array}$ & $\begin{array}{c}\text { Mes de } \\
\text { gestación }\end{array}$ \\
\hline 15 & Sec. incomp. & Soltera & Puno & Noveno \\
\hline 16 & Sec. comp. & Conviviente & Lima & Séptimo \\
16 & Sec. comp. & Conviviente & Lima & Octavo \\
\hline 17 & Sec. comp. & Conviviente & Lima & Noveno \\
\hline 17 & Sec. comp. & Conviviente & Lima & Octavo \\
\hline 17 & Sec. incomp. & Conviviente & Lima & Quinto \\
\hline 17 & Téc. incomp. & Conviviente & Lima & Noveno \\
\hline 18 & Sec. comp. & Conviviente & Lima & Sétimo \\
19 & Sec. comp. & Conviviente & Ancash & Sexto \\
\hline 19 & Téc. incomp. & Conviviente & Lima & Noveno \\
\hline 19 & Sec. comp. & Soltera & Lima & Octavo \\
\hline 19 & Prim incomp. & Conviviente & Cajamarca & Séptimo \\
19 & Sec. comp. & Conviviente & Lima & Sexto \\
\hline 19 & Sec. incomp. & Soltera & Ucayali & Octavo \\
\hline \multicolumn{7}{c}{} \\
\hline
\end{tabular}

\section{PROCEDIMIENTOS}

En un primer momento, y con el permiso del Director del Hospital, se realizó la observación directa de la interacción entre la joven embarazada y la obstetriz en el triaje obstétrico, en el servicio de psicoprofilaxis, en la consulta con el médico ginecoobstetra general y de la especialidad del adolescente, con el objetivo de profundizar en el análisis de la información preliminar, los cuales fueron recabados siguiendo un guión de observación directa y anotados en fichas de campo.

Luego de ello se complementó la guía para las entrevistas a profundidad, la cual presentaba preguntas flexibles. Las jóvenes que aceptaron participar del estudio, fueron entrevistadas en un ambiente del hospital diferente a un consultorio, que brindaba un ambiente de tranquilidad y de encuentro cercano con las entrevistadas. El personal 
de salud fue entrevistado en sus consultorios. En ambos grupos se procedió a la grabación de la entrevista previa autorización.

Finalmente, se realizó un grupo focal con doce gestantes en el centro juvenil del Hospital, para evaluar aspectos que faltaban ser explorados en la observación directa y las entrevistas a profundidad, entre los que se incluyó: evaluación de la atención recibida en el control prenatal, percepción y expectativas del control prenatal, vivencias del proceso del embarazo, aspectos que definían la gestación, problemas y beneficios del control prenatal, riesgos y temores en el control prenatal.

Procedimos, como facilitadora, a llevar la dinámica de grupos focales, luego les planteamos las preguntas de la guía, promoviendo una conversación y discusión espontánea. Posteriormente, se entregó papelógrafos, tarjetas y plumones, a fin de que plasmaran sus ideas. Luego pegamos los papelógrafos y tarjetas en una pizarra acrílica, socializando las opiniones, propuestas y observaciones de las participantes; propiciamos el intercambio de ideas y discusión. Se usó una grabadora para recoger los detalles de la información. No se pudo efectuar una dinámica de grupo focal con el personal de salud, en tanto no hubo acuerdo con relación al horario.

\section{ASPECTOS ÉTICOS}

El estudio fue revisado y aprobado por el Comité de Ética de la Universidad Peruana Cayetano Heredia y del Hospital Nacional donde se realizó el estudio, a sugerencia de ellos se obvia el nombre del Hospital. Se usó consentimiento informado para el personal de salud, gestantes mayores de edad y las madres que acompañaban a las gestantes menores de edad, y asentimiento informado para las menores de edad. Se solicitó su autorización para grabar las conversaciones, así como para observar la interacción entre gestantes y personal de salud durante las consultas. Los nombres que se consignan en los resultados son referenciales, más no reales.

\section{ANALISIS DE LA INFORMACIÓN}

La información cualitativa fue analizada con la ayuda del software Atlas/Ti versión 2,4, desde las categorías analíticas del significado de la sexualidad, el embarazo y el control prenatal, que luego fueron exploradas en mayor grado en el grupo focal a las jóvenes embarazadas.

Para fundamentar el abordaje y tratamiento de los datos, se tomó en cuenta: 1) El interaccionismo simbólico (11), que propone aproximarse a la interpretación sociocultural a través del estudio de la interacción entre los individuos;
2) la teoría de la comunicación humana ${ }^{(12)}$, plantea que no solo transmite información, sino que a la vez, influye mutuamente en la correspondencia entre los individuos; y 3) el modelo médico hegemónico (13) según el cual en la relación médico-paciente existe subordinación y pasividad en la interacción entre individuos. En ese sentido, se prioriza el control ideológico y técnico en la práctica médica, en torno al saber/no saber, en la que el que "sabe" utiliza un lenguaje científico, verificado y universal con relación a otros "saberes que son considerados como tales".

\section{RESULTADOS}

En estos resultados nos referimos a la sexualidad y la comunicación desde las dificultades que tiene el personal de salud y las jóvenes embarazadas para establecer una interacción en el control prenatal. Asimismo, nos remitimos a la manera cómo el personal de salud ha comprendido el embarazo en la adolescencia, refiriéndonos a los actores que intervienen en la responsabilidad de este.

Del mismo modo, vemos la percepción que tiene el personal de salud, respecto al embarazo, a partir del control del cuerpo como medio a través del cual determinan el estado de salud o enfermedad en la gestante adolescente; haciendo alusión al papel que tienen las obstetrices en la preparación del parto.

Con respecto al trato durante el control prenatal señalamos la interacción diferenciada entre el personal de salud femenino/masculino y las jóvenes embarazadas; asimismo, referimos formas de comunicación verbal y no verbal de parte del personal de salud médico, que advierten problemas en la comunicación.

\section{SEXUALIDAD Y COMUNICACIÓN}

El personal de salud resaltaba el papel de los medios de comunicación social en la orientación de la familia en el tema de la sexualidad y prevención de la gestación en la adolescencia.

...Los medios de comunicación no toman las medidas necesarias para que la familia tenga una preparación necesaria, porque tienden a dar otra cosa, entretenimiento y no realizan una buena comunicación para aprender a identificar los riesgos de embarazarse durante la adolescencia. (Ginecoobstetra).

Además, la Jefa del Servicio de Ginecoobstetricia del Adolescente, afirmaba que trataba de explicarles a las 
jóvenes embarazadas, sobre todo si son menores de quince años, los riesgos que significa un embarazo en la adolescencia, pero que siempre descubría que estas no habían culminado su nivel de instrucción.

...La médica pregunta a la gestante ¿hasta qué año estudiaste? La joven embarazada responde: estaba terminando quinto. La médica vuelve a preguntar: ¿tu embarazo fue con tu enamorado? Sí, responde la gestante adolescente. La médica comenta: lo primero que hago es preguntarles hasta qué año estudiaron, la mayoría tiene secundaria incompleta. Si se embarazaron con su enamorado; y el perfil de las gestantes siempre es ese $y$ tú dices, esas son las condiciones que se dan y la base es la educación (Ginecoobstetra de adolescentes).

Durante el control prenatal los ginecoobstetras generales solicitaban a las jóvenes embarazadas su colaboración en los exámenes clínicos, señalando que deberían hacerlos por el bienestar del bebé.

El médico solicita a la gestante que se haga una prueba para saber si el parto será normal o por cesárea. Después le pide que suba a la camilla y para examinarla utiliza su instrumental para la prueba de Papanicolaou y la gestante adolescente se queja de dolor. El médico le dice: "me molesto...tienes que colaborar con el médico es tu obligación por el bien de tu bebé" (Ficha de observación).

Las jóvenes embarazadas expresaron que los ginecoobstetras les manifestaban su desacuerdo por no haber prevenido el embarazo en su adolescencia.

...El doctor me preguntó cómo me siento, cosas así...me preguntó si el bebé se movía y yo le dije no tanto. Luego me dijo también que no me había cuidado, que estaba muy jovencita... (Johana; 17 años).

\section{RESPONSABILIDAD DEL EMBARAZO: ENTRE LA MADUREZ Y LA EMOCIÓN}

Las obstetrices del servicio de consejería señalaban que las jóvenes embarazadas tenían poca responsabilidad en el embarazo, dado que continuaban siendo adolescentes y dejaban al hospital la responsabilidad de atenderlas.

...El cuidado que las gestantes le atribuyen a su cuerpo no depende de su nuevo estado, ella sigue siendo adolescente. Ella asume el embarazo como si tuviera un lunar, está embarazada pero no se preocupa totalmente, como todas las personas que vienen a un hospital le atribuyen la responsabilidad de su cuidado al lugar al que vienen (Obstetriz de consejería del adolescente).

Además, el personal de salud resaltaba la falta de relación entre lo biológico y social en las jóvenes embarazadas, y el proceso de madurez física en ellas.

...La última de trece años que vino, era una adolescente mucho más alta que yo, aparentaba de veinte años pero, tenía trece. Es que las niñas tienen una edad psicológica y social diferente y me apena porque ellas no saben enfrentar (Ginecoobstetra de adolescentes).

Con respecto a la responsabilidad del embarazo, el personal obstétrico afirmaba que el centro de salud $u$ hospital no era el más indicado para informar sobre sexualidad a las jóvenes gestantes, que debería hacerlo la escuela desde los primeros años de edad, teniendo en cuenta que en la familia existen barreras que no permiten comprender la sexualidad.

... Aún parece que todas las familias no están listas, o sea no están mirando ahí...y pensando que la niña es un ser limitado, mientras las niña ya está volando y pensando en otra cosa....muchas de las niñas que ahora están embarazadas tienen el enamorado desde los nueve años, imagínate cómo hacían para verlo si a esa edad estaban en su casa y no la dejaban salir. Y por qué no se dan cuenta los padres de lo que pasaba...no están listos para afrontar los cambios. (Ginecoobstetra de adolescentes).

\section{EL CONTROL DEL CUERPO EN LA ATENCIÓN PRENATAL}

Los residentes varones del servicio de ginecoobstetricia destacaban que el cuerpo de las jóvenes embarazadas era un medio para llegar a la enfermedad en términos biomédicos.

... Para nosotros, conocer el cuerpo de las gestantes adolescentes representa en lo que es la parte ginecológica, el cuerpo humano, simplemente buscar la patología orgánica y llegar a un diagnóstico. (Residente varón ginecoobstetra).

Además, los residentes varones del servicio de ginecoobstetricia general, señalaban que existe muchas veces temor en las jóvenes embarazadas durante el parto, debido a que la información que recibían de parte del personal obstétrico en el servicio de psicoprofilaxis era teórica. 
...El temor que he podido percibir no es tanto durante la consulta médica sino, durante el inicio de la labor del parto, durante las primeras contracciones, porque a veces llegan sin haber recibido una charla adecuada acerca de sus temores....Asistir a la psicoprofilaxis puede disminuir el temor al dolor, pero muchas veces la charla es una ayuda teórica. (Residente varón ginecoobstetra).

De otro lado, las jóvenes embarazadas indicaban que cuando mostraban su cuerpo a los ginecoobstetras por primera vez, sentían vergüenza de ello, pero toleraban está situación por el bien de su bebé.

...Cuando tuve que mostrar mi cuerpo al doctor por primera vez, me sentí avergonzada e incómoda, porque estoy descubriendo mi cuerpo y mostrándolo a una persona que no conozco...pensaba en lo que me decía mi mamá, que era el trabajo del doctor, que no me iba a ver con ojos de hombre, sino como doctor. (Carmen; 17 años).

En cambio, las jóvenes embarazadas manifestaban que cuando eran atendidas la primera vez, por el personal de salud femenino no sentían tanta vergüenza.

A pesar de que me atendía una médica, sentía un poco de vergüenza, porque su cuerpo es casi igual al mío, no siento que me está viendo con otros ojos (Leticia; 16 años).

Para el personal de salud, la concepción de riesgo como adquirir las ETS y el VIH/SIDA y complicaciones del embarazo en la adolescencia, siempre estaban presentes. Las enfermedades infecciosas se debían, según los proveedores de salud, a la vida sexual activa que mantenían las jóvenes embarazadas.

...El embarazo no es lo peor que les haya pasado, sino lo peor es tener alguna enfermedad porque están expuestas a eso, las parejas que tienen no son estables, no tienen una sola pareja sexual. (Obstetriz del servicio de consejería).

\section{EL TRATO EN EL CONTROL PRENATAL}

Los ginecoobstetras generales no mantenían la mirada durante la consulta prenatal con las jóvenes embarazadas, sino que escribían constantemente en la historia clínica.

El ginecoobstetra general pregunta a la gestante adolescente: ¿edad? ¿última regla? ¿se hizo la prueba de Papanicolaou?...la joven responde rápidamente... (Ficha de observación).
Además, las jóvenes embarazadas narraban que los médicos del Hospital Nacional de Lima, durante la consulta prenatal les manifestaban su precocidad sexual en la adolescencia.

El doctor me preguntó si se movía el bebé y me dijo ique no me había cuidado! que estaba muy jovencita... (Rosalía; 17 años).

Las jóvenes gestantes señalaban que preferían recibir un trato afectuoso y dialogante del médico ginecoobstetra general, como el que observaban en las obstetrices del Hospital Nacional de Lima.

Me gusta que me traten con cariño, me da más confianza y mejor trato para conversar con el doctor. Me siento más a gusto para conversar con una persona.....la señorita es bien cariñosa, es bastante amorosa...los médicos no son así, jellos te dicen señora!" (Carmen Rosa; 19 años).

Asimismo, las jóvenes embarazadas destacaban que durante el trato en la consulta prenatal, el personal de salud no les daba explicaciones claras sobre los términos que utilizaba para hacer el diagnóstico de su embarazo.

¿Le preguntaste al doctor a qué se refería con normal? Sí, pero no me contestó completamente, solo me dijo que me subiera a la camilla. Como que no me prestaba atención y no me daba confianza para conversar sin molestarse... Me decía que todo estaba bien, ipero no me explicaba nada! (Leticia; 16 años).

El médico solicita a la joven embarazada que suba a la camilla para escuchar los latidos del bebé $y$ medir el vientre. Además le dice que debe estimular los pezones. La embarazada responde que lo hace todos los días y el médico lo dice que le está engañando....que debe hacer eso, para que bajen las contracciones y pueda dar a luz... ise entendió bien! (Ficha de Observación).

Las jóvenes embarazadas consideran que las prescripciones médicas y el "tratamiento" son positivos, en términos de bienestar y salud para ellas y sus bebés. Sin embargo, cabe señalar que cuando estos no se brindan, las jóvenes observan que obedece a la ausencia de explicaciones del personal de salud frente a sus necesidades de información; por eso, siguen los consejos de sus familiares, independientemente de lo dicho por el médico.

... El doctor me dijo que todavía no iba a dar a luz, le pregunté por qué, me dijo que jtodavía no! Cuando lle- 
gué a mi casa le conté a mi abuelita y preparó una tina con yerbas para que yo me bañara ahí, pudiera dar a luz pronto y de manera natural... (María; 19 años).

En ese sentido, las jóvenes embarazadas deseaban recibir una mejor atención de parte del personal de salud, que las ayudase a conocer el proceso de crecimiento del bebé en sus vientres.

...A mí me gustaría que me recomienden y orienten sobre algunas cosas más...que me mostraran cómo va creciendo un bebé dentro de mí cuerpo (Carmen; 16 y grupo focal).

Además, para las jóvenes embarazadas, ser llamadas por sus nombres, significaba ser tratadas con confianza, sentirse bien y no ser consideradas una joven más durante la atención prenatal.

Me gusta que me llamen por mi nombre, que me traten con cariño, me da más confianza, mejor trato para mí. Me siento más a gusto para conversar con una persona... (Sofía;16 años).

... Me da confianza y me permite sentirme bien... se siente bien que no te traten como una chica más, sino como una chica que necesita que la ayuden, que la orienten (Sofía;16 años).

\section{DISCUSIÓN}

Para el personal de salud el rol de la familia estaba muy presente en la orientación de las jóvenes sobre la sexualidad en la adolescencia. Sin embargo, en algunos estudios plantean ${ }^{(14)}$ que las adolescentes prefieren que los centros de salud sean el principal canal de apoyo en su adolescencia, esperando que estos sean amigables y brinden información adecuada y completa en dicha etapa.

No obstante, en la atención prenatal, la demanda de las jóvenes embarazadas primerizas se centra en conocer el proceso de su embarazo y afectividad en este período. Pero, no encuentra respuesta de parte del personal de salud, en la medida en que excluye la posibilidad de asumir una actitud pedagógica que aporte al conocimiento y crecimiento personal de la joven embarazada, desde que se asume que por lo general, ellas tienen un bajo nivel cultural, otorgándoles con esto un papel receptivo, poco espacio para aclarar sus dudas, necesidades y preocupaciones; subestimando su capacidad de entendimiento en los procesos de su gestación durante la adolescencia, como lo señalan Luengo ${ }^{(15)}$ y Magallanes ${ }^{(16)}$.
De esa manera, hemos observado que durante la atención prenatal el personal de salud, enfatiza más en el control del cuerpo de las jóvenes embarazadas, destacando los aspectos biomédicos, en la medida en que dan prioridad a lo que se tiene que expresar y registrar el diagnóstico clínico, que poner en práctica habilidades mínimas para escuchar o dialogar con las jóvenes embarazadas. En ese contexto, Watzlawick ${ }^{(12)}$ señala, que la comunicación o interacción social no solo transmite información, sino que a la vez influye mutuamente en la definición de la relación entre los individuos. Involucrando en ello, dos procesos: la comunicación digital (lo que se dice, el mensaje) y la comunicación análoga (cómo se dicen las cosas, la metacomunicación).

En ese sentido, durante el control prenatal, el personal de salud masculino, no mantenía "la mirada" hacia las jóvenes embarazadas; sino que escribía constantemente en la historia clínica. Expresando con ello indiferencia y distancia en la interacción, que en los términos de Watzlawick, significa transmitir comunicación en la interacción, a través de gestos y movimientos corporales, que en todo caso cuando se expresa a través de comunicación verbal, permite un monólogo pero no un diálogo durante la consulta entre el personal de salud y las jóvenes embarazadas como lo resaltan Rubín de Celis y Vidal ${ }^{(17)}$

En referencia al trato durante el control prenatal, cabe destacar que existen importantes diferencias de género en la interacción médico, médica, obstetriz y joven embarazada, que generan consecuencias desfavorables en la comunicación y seguimiento de recomendaciones médicas y obstétricas. En ello también observamos que el personal de salud femenino brinda mayor confianza, tranquilidad y calidez a la joven embarazada durante el control prenatal ${ }^{(18,19)}$. En cambio, el proveedor de salud masculino, es más controlador y técnico. Al respecto Aguirre ${ }^{(20)}$ afirma que existen diferencias de género en el trato durante la atención prenatal. Siendo la interacción entre las jóvenes embarazadas y el personal de salud femenino más afectivo y social. Y entre estas y el personal de salud masculino, generalmente, cognitivo y operativo.

Para las jóvenes gestantes los significados del control prenatal inciden en la observación, intervención y control de su cuerpo en la interacción. Sin embargo, para ellas, esto significa un proceso de aprendizaje que, les permite descubrir su identidad como madres, asegurando el bienestar de su bebé ${ }^{(21,22)}$.

En conclusión, las jóvenes embarazadas tienen la necesidad de mantenerse informadas durante el proceso del embarazo. Sin embargo, en el personal de salud existe la tendencia a no brindarles explicaciones relativas 
al crecimiento del bebé y nutrición desde que se asume que el bajo nivel cultural de ellas, no facilita una adecuada interacción, privilegiándose la información sobre riesgos biomédicos.

Las jóvenes embarazadas y el personal de salud tienen una interacción diferenciada en cuanto al trato que brindan en la atención durante el control prenatal. El personal de salud femenino es afectivo y orientador en la relación con las jóvenes mientras que el personal de salud masculino interacciona de manera operativa y cognitiva.

\section{AGRADECIMIENTOS}

A Tanya Taype por su asesoría en el curso de esta investigación.

\section{Fuente de Financiamiento}

Autofinanciado.

\section{Conflictos de Interés}

La autora declara no tener conflictos de interés en la publicación de este artículo.

\section{REFERENCIAS BIBLIOGRÁFICAS}

1. World Health Organization. Adolescent pregnancy. Issues in adolescent health and development. Geneva: WHO; 2004.

2. Coll A. Embarazo en la adolescencia ¿cuál es el problema? En: Donas S (comp). Adolescencia y juventud en América Latina. Cartago: Libro Universitario Regional; 2001. p. 42748.

3. World Health Organization. Maternal mortality in 2005: estimates developed by WHO, UNICEF, UNFPA, and the World Bank. Geneva: WHO; 2007.

4. Ronsmans C, Graham WJ, Lancet Maternal Survival Series steering group. Maternal mortality: who, when, where, and why. Lancet. 2006;368(9542):1189-200.

5. Seclen-Palacín JA, Jacoby E, Benavides B, Novara J, Velásquez A, Watanabe $\mathrm{E}$, et al. Efectos de un programa de mejoramiento de la calidad en los servicios maternos perinatales en el Perú: La experiencia del Proyecto 2000. Rev Bras Saude Matern Infant. 2003;3(4):421-38.

6. Simkhada B, Teijlingen ER, Porter EM, Simkhada P. Factors affecting the utilization of antenatal care in developing countries: systematic review of the literature. J Adv Nurs. 2008;61(3):244-60

7. Beltrán A. Determinantes de la utilización de servicios de control del embarazo y parto: el caso peruano. En: Cortez $\mathrm{R}$ (ed). Salud, equidad y pobreza en el Perú, teorías y nuevas evidencias. Lima: Centro de Investigación de la Universidad del Pacífico; 2002. p. 415-56.

8. Hurtado R, Ramos M. Perfil de salud de las mujeres y los hombres en el Perú 2005. Lima: Organización Panamericana de la Salud; 2006.
9. Valverde R. Percepciones y comportamientos frente a usuarias adolescentes. En: Cordero M, Jiménez O (ed). Más allá de la intimidad cinco estudios en sexualidad, salud sexual y reproductiva. Lima: Pontificia Universidad Católica del Perú; 1996. p. 89-107.

10. Jasis M. A palabras de usuaria ... ¿oídos de doctor?. Una exploración sobre las necesidades de salud reproductiva de las mujeres en La Paz, Baja California. En: Stern C, Echarri CJ (coord). Salud reproductiva y sociedad. Resultados de investigación. México DF: El Colegio de México; 2000. p. 97-143.

11. Blumer H. Symbolic interactionism. Perspective and method. New Yersey: Prentice-Hall; 1969.

12. Watzlawick P, Helmick, J, Jackson, D. Teoría de la comunicación humana. Interacciones, patologías y paradojas. Buenos Aires: Editorial Tiempo Contemporáneo; 1972.

13. Menéndez E. Antropología médica, orientaciones, desigualdades y transacciones. México DF: Centro de Investigaciones y Estudios Superiores en Antropología Social; 1990.

14. Quintana A, Hidalgo, C, Dourojeanni D. Escuchen nuestras voces. Representaciones sociales e itinerarios de salud sexual y reproductiva de adolescentes y jóvenes. Lima: Instituto de Educación y Salud; 2003.

15. Luengo $X$. Aspectos éticos en la atención de adolescentes y en la prevención del embarazo no deseado. En: Arana MT, Calle MC, Arana M (ed). Promoción y cuidado de la salud de adolescentes y jóvenes: Haciendo realidad el derecho a la salud. Lima: OPS, GTZ, SPAJ; 2004

16. Magallanes M. Significados sociales del embarazo y uso de los servicios prenatales: un estudio cualitativo realizado en el Hospital Hipólito Unanue de Lima, Perú. [Tesis para optar el Grado de Magíster en Salud Pública]. Lima: Universidad Peruana Cayetano Heredia; 2001.

17. Rubín de Celis E, Vidal, E. Relaciones sociales en la atención de salud: Rompiendo paradigmas. Fascículo 3. Lima: Universidad Peruana Cayetano Heredia; 2005.

18. Pérez C, Pastene C, Carrasco B, Mora C, Grau M, Rodríguez M. Mirada al gineco-obstetra en la atención ambulatoria. Rev Chil Salud Publica. 2003;7(2):74-79.

19. Matamala MI. Calidad de atención en las mujeres desde la perspectiva de género. Santiago de Chile: Instituto Chileno de Medicina Reproductiva; 1994.

20. Aguirre Baztán Á. Etnografía: metodología cualitativa en investigación sociocultural. Mexico DF: Alfaomega; 1995.

21. Bustamante I. Significados del embarazo y la maternidad en la construcción de sus identidades en adolescentes de 15 a 19 años de sector socio-económico bajo, que residen en la ciudad de Lima. [Tesis para optar el Grado de Magíster en Salud Pública]. Lima: Universidad Peruana Cayetano Heredia; 2000

22. Buitrón A. Identidad y maternidad: estereotipos de género, maternidad adolescente y barreras ante la planificación familiar. Lima: Asociación Peruana para la Salud Mental para la Mujer; 2003.

Correspondencia: Carolina Blossiers Mazzin

Correo electrónico: blossiers mazzini@yahoo.es 